\title{
Comparative study of foreign visitors' perceptions of places by analyzing visual content of photographs
}

\author{
Bochra BETTAIEB $^{\text {a }}$, Yoshiki WAKABAYASHI ${ }^{\mathrm{b}}$ \\ ${ }^{a}$ Tokyo Metropolitan University, bochra_bettaieb@hotmail.fr \\ ${ }^{b}$ Tokyo Metropolitan University, wakaba@tmu.ac.jp \\ * Corresponding author
}

Keywords: Flickr, photo image, machine learning, spatial analysis, Tokyo

\begin{abstract}
:
Photographs taken by tourists have the potential to play an important role in understanding visitors' behaviors and perceptions in tourist destinations. In recent years, a variety of studies have been conducted to use geotagged photographs contained in social media to capture the digital footprint of human spatial behavior. The authors (Bettaieb and Wakabayashi 2021) had analyzed geocoded photographs taken in central Tokyo to compare the behavior patterns of foreign visitors. However, our previous study could not identify the content of photographs that enable us to capture visitors' perceptions of places. To resolve this issue, the present study applied a machine-learning method to classify the visual content of photographs. In particular, we focused on differences according to visitors' home countries.

The data obtained were geocoded photos taken within $1.5 \mathrm{~km}$ from three stations located in three major tourism sites of Tokyo: Shinjuku, Asakusa, and Ginza. We used 5,446 photos taken by visitors to Tokyo from four major countries, the United States, the United Kingdom, Taiwan, and Singapore, who posted their pictures on Flickr in 2018. The images of photographs were analyzed by using Google Cloud's Vision API, which enables us to assign labels to images and automatically classify them into predefined categories. On the basis of the labels assigned to the photographs, we manually classified them into 11 categories. By employing the locational information of Flickr data, we geocoded each photograph as an input to GIS. The spatial distribution of photos that indicate the areas of interest (AOIs) for visitors was compared according to countries of residence of visitors by employing spatial analysis methods to capture the variation in perception of places.

Comparison of the distribution of the category of photos proved the differences in how three tourism sites are perceived by visitors: Shinjuku is characterized by modern urbanism and popular culture; Asakusa is characterized by traditional culture, urbanism, and people; and Ginza is mainly characterized by modern urbanism. These differences were confirmed by the spatial distribution of AOIs for visitors. These results indicate that visitors' differing perceptions of places are explained by the spatial structure and attractions of tourism sites.

Concerning differences according to country of visitors' residence, such differences were evident in Shinjuku and Ginza, while visitors to Asakusa showed a common perception of place characterized by traditional culture and urbanism. Analysis of the spatial distribution of photos by visitors' home country revealed that American visitors in Shinjuku and British visitors in Ginza showed a distinctive feature of place perception closely related to the deviation of the spatial distribution of AOIs. In Asakusa, few differences were observed in spatial patterns of AOIs for visitors according to home countries. The reason for this is that tourist attractions are concentrated in and around specific facilities in the Asakusa district. Thus, we could show the variation of visitors' perceptions of places according to their home countries by employing an image analysis method and GIS.
\end{abstract}

\section{References}

Bettaieb, B. and Wakabayashi, Y. 2021. Comparison of the areas of interest in central Tokyo among visitors by country of residence using geotagged photographs. Geographical Review of Japan Series B 93(2): 66-75. 